\title{
miR-218-5p restores sensitivity to gemcitabine through PRKCE/MDR1 axis in gallbladder cancer
}

\author{
Hui Wang ${ }^{1,4}$, Ming Zhan ${ }^{1,4}$, Sun-Wang $\mathrm{Xu}^{1,4}$, Wei Chen ${ }^{1}$, Man-Mei Long ${ }^{2}$, Yong-Heng Shi ${ }^{2}$, Qiang Liu $^{2}$, Man Mohan ${ }^{3}$ and Jian Wang ${ }^{*, 1}$
}

Gallbladder cancer (GBC) is one of the most common malignancy of the biliary tract characterized by its high chemoresistant tendency. Although great progresses have been made in recent decades for treating many cancers with anticancer drugs, effective therapeutics methods for anti-GBC are still lacking. Therefore, investigations into identifying the mechanisms underlying the drug resistance of GBC are greatly needed. In this study, we show that miR-218-5p plays a critical role in gemcitabine resistance of GBC. miR-218-5p levels were significantly lower in GBC than adjacent non-cancer tissues, and which were also associated with patient prognosis. While miR-218-5p overexpression abrogated gemcitabine resistance of GBC cells, silencing of which exhibited the opposite effects. Via six microRNA targets prediction algorithms, we found that PRKCE is a potential target of miR-218-5p. Moreover, miR-218-5p overexpression repressed the luciferase activity of reporter constructs containing $3^{\prime}-U_{T R}$ of PRKCE and also reduced PRKCE expression. Further studies revealed that miR-218-5p promotes sensitivity of gemcitabine by abolishing PRKCE-induced upregulation of MDR1/P-gp. Taken together, our results imply that an intimate correlation between miR-218-5p and PRKCEIMDR1 axis abnormal expression is a key determinant of gemcitabine tolerance, and suggest a novel miR-218-5p-based clinical intervention target for GBC patients.

Cell Death and Disease (2017) 8, e2770; doi:10.1038/cddis.2017.178; published online 11 May 2017

Gallbladder cancer (GBC) accounts for about $40 \%$ of all biliary tract carcinomas, and is one of the most common malignancy of the biliary tract. ${ }^{1,2}$ Although associated with poor prognosis, the mechanism underlying the aggressive malignancy of GBC remains largely unknown. Moreover, unlike several other tumors, it is highly resistant to the currently available standard adjuvant therapy, which makes its treatment even more challenging. ${ }^{3}$ Although several potential targets and signaling pathways underlying GBC chemoresistance have been revealed, the precise mechanisms are still elusive. ${ }^{4-6}$ Thus deeper unraveling the molecular mechanisms of drug resistance is of vital importance and urgently needed.

MicroRNAs (miRNAs), 22 nucleotides on average, are a group of evolutionarily conserved, small, endogenous, singlestranded non-coding RNAs. ${ }^{7}$ Through binding the 3 '-untranslated regions ( $3^{\prime}$-UTRs) of their target genes, they could regulate specific genes expression at the posttranscriptional level. Multiple miRNAs have been found implicated in various kinds of cancers. miRNAs are capable of modulating cell proliferation, differentiation, metabolism, apoptosis, and thus actively participate in regulating tumorigenesis and tumor progression. ${ }^{8,9}$ Evidence suggests that miRNA can modulate the therapeutic efficacy in several cancer types, ${ }^{10}$ but it is still unclear whether and how deregulated miRNAs are involved in the chemoresistance of GBC. miR-218-5p, a tumor suppressor miRNA, has been found to be downregulated in several cancer types such as GBC, cervical cancer, colon cancer, and prostate cancer. ${ }^{11-14}$ In this study, we assessed the involvement of miR-218-5p in GBC chemosensitivity.

Permeability glycoprotein (P-gp), also known as multidrug resistance 1 (MDR1), is a well-known membrane transporter, which is able to transport various kinds of toxic substances and exerts a protective effect under physiological conditions. However, this function when utilized by cancer cells, may lead to the effluxing of many drugs and enhanced chemoresistance. ${ }^{15}$ PRKCE, a member of protein kinase C (PKC) family, is a serine- and threonine-specific protein kinase that actively participate in promoting drug resistance through phosphorylating a variety of protein targets, such as P-gp, ATF2, PI3K, Stat3, and Erk. ${ }^{16-17}$

Here, using a genome-wide miRNA expression profiling in six pairs of GBC and the corresponding non-cancerous gallbladder (CNG) tissues, we found that miR-218-5p is significantly downregulated in GBC. Further, we show that miR-218-5p regulates gemcitabine sensitivity in GBC cells by simultaneously repressing $P R K C E$ expression. Due to the ability of PRKCE to activate MDR1/P-gp, repression of miR-218-5p causes abolishment of PRKCE inhibition and leads to increased MDR1/P-gp levels, and then enhances gemcitabine resistance of GBC cells. Moreover, we show that miR-218-5p and PRKCE are prognostic markers in GBC patients receiving chemotherapy. This adds to the possibility that strengthening of GBC chemoresistance might be countered by restoring expression of miR-218-5p, a notion that can be tested in the clinic.

\footnotetext{
${ }^{1}$ Department of Biliary-Pancreatic Surgery, Renji Hospital, School of Medicine, Shanghai Jiao Tong University, Shanghai, China; ${ }^{2}$ Department of Pathology, Renji Hospital, School of Medicine, Shanghai Jiao Tong University, Shanghai, China and ${ }^{3}$ Department of Biochemistry and Molecular Cell Biology, Shanghai Key Laboratory of Tumor Microenvironment and Inflammation, Institutes of Medical Sciences, Shanghai Jiao Tong University, School of Medicine, Shanghai 200025, China

${ }^{*}$ Corresponding author: J Wang, Department of Biliary-Pancreatic Surgery, Renji Hospital, School of Medicine, Shanghai Jiao Tong University, 160 Pujian Road, Shanghai 200127, China. Tel: +86 21 68383990; Fax: +86 21 58394262; E-mail: dr_wangjian@126.com

${ }^{4}$ These authors contributed equally to this work.

Received 26.11.16; revised 03.3.17; accepted 08.3.17; Edited by P Agostinis
} 


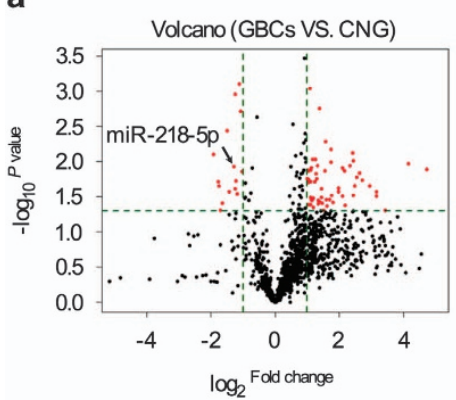

d

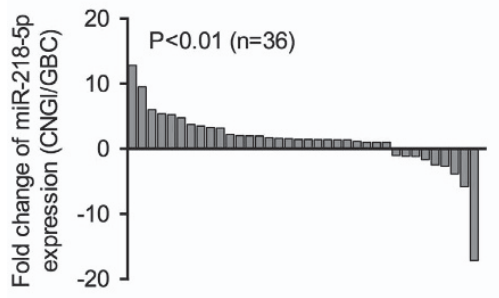

g

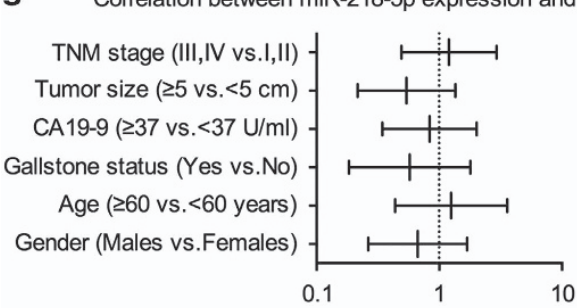

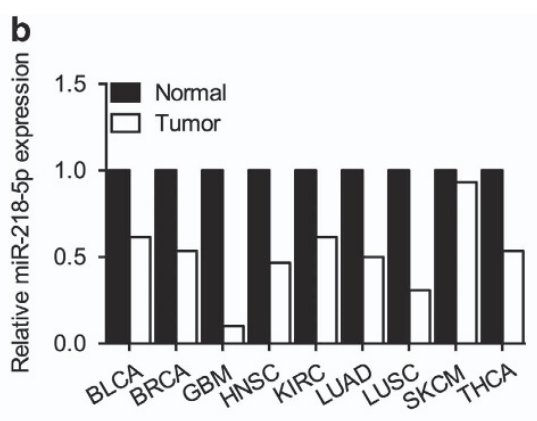
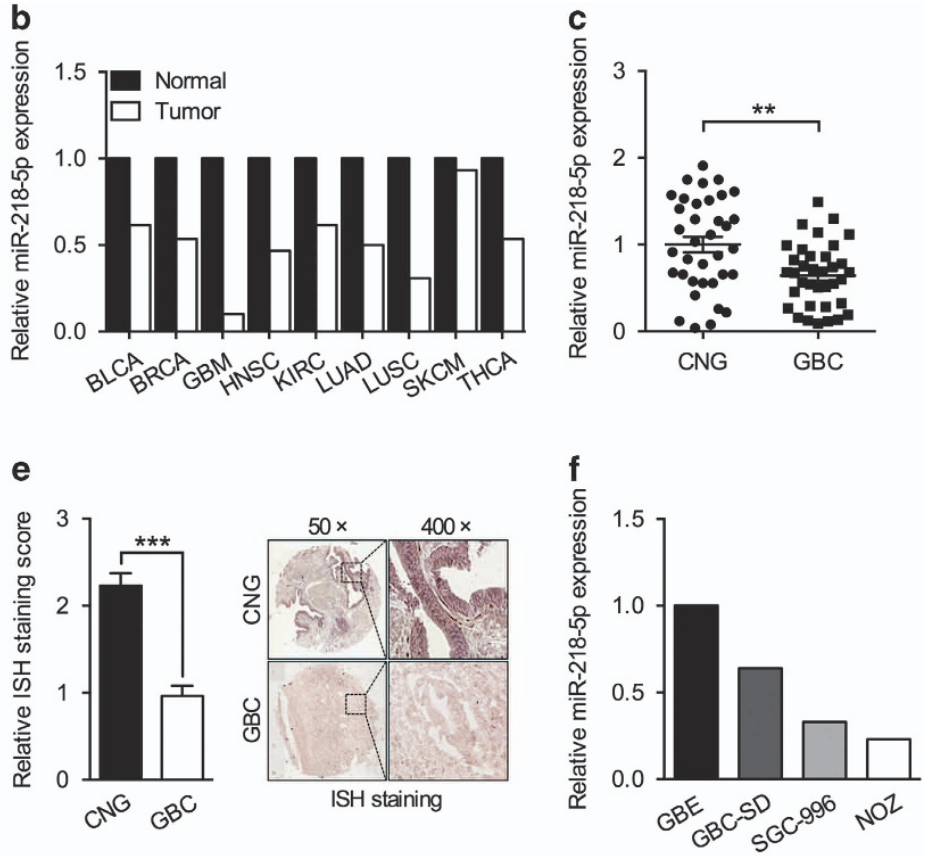

Correlation between miR-218-5p expression and clinic characteristics

\begin{tabular}{cc}
$1.20(0.49-2.93)$ & 0.69 \\
$0.54(0.22-1.35)$ & 0.19 \\
$0.83(0.34-2.01)$ & 0.68 \\
$0.57(0.18-1.79)$ & 0.34 \\
$1.25(0.44-3.58)$ & 0.68 \\
$0.67(0.26-1.68)$ & 0.39 \\
\hline$H R(95 \% C l)$ & $P$ value
\end{tabular}

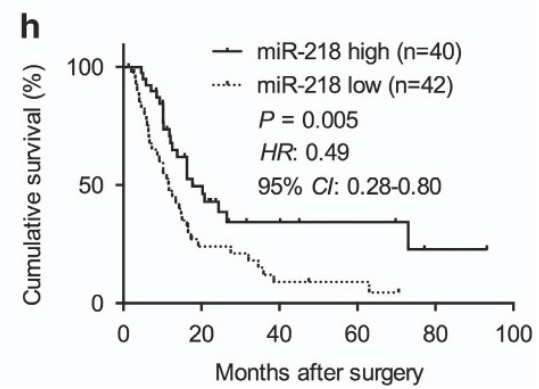

Figure 1 miR-218-5p is downregulated in GBC tissues and cell lines, and is associated with prognosis of GBC patients. (a) Volcano plot of microRNAs expression array from six pairs of GBC and CNG tissues. More detailed information can be found in Supplementary Materials. (b) miR-218-5p expression in other nine types of cancer from starBase v2.0 Pan-cancer Project database. (c) Validation of miR-218-5p differential expression in an independent cohort contained 36 pairs of GBC and CNG tissues. (d) Fold change of miR-218-5p expression in new 36 pairs of GBC and CNG tissues. (e) Comparison of miR-218-5p expression in 82 pairs of GBC and CNG FFPE tissues (left) and their representative ISH image stained by anti-miR-218-5p probe (right). (f) Relative expression of miR-218-5p in GBC cells and GBE cells. (g) Comparing different TNM stages, tumor size, CA19-9 level, gallstone status, age and gender ratio between miR-218-5p high and miR-218-5p low expression from 82 pairs of GBC FFPE tissues. Statistical significance was performed by the $\chi^{2}$-test. (h) Kaplan-Meier analysis of the correlation between miR-218-5p expression and POS in 82 GBC patients. Small nuclear RNA U6 was used to normalize the Q-PCR results. bar, S.E.M., ${ }^{\star \star} P<0.01$; ${ }^{* \star} P<0.001$; Student's $t$-test. BLCA, urothelial bladder cancer; BRCA, breast cancer; GBM, glioblastoma multiforme; HNSC, head and neck squamous cell carcinoma; KIRC, clear cell kidney carcinoma; LUAD, lung adenocarcinoma; LUSC, lung squamous cell carcinoma; SKCM, cutaneous melanoma; THCA, papillary thyroid carcinoma

\section{Results}

Reduced miR-218-5p expression in GBC well correlated with tumor prognosis. To identify transcripts that potentially drive malignance of GBC, a miRNA expression profile was determined by microarray analysis. The volcano plot and heat map showed systematic variations in transcript expression levels of miRNAs between GBC tissues and CNG tissues from six GBC patients (Figure 1a, Supplementary Figure 1, and Supplementary Table 1). Further analyzing via our miRNA expression profile data and other microarray databases (starBase v2.0; http://starbase.sysu.edu.cn/) of different tumors, ${ }^{18}$ we found that miR-218-5p is one of the common target reduced in many kinds of tumors (Figure $1 \mathrm{~b}$ and Supplementary Figure 2). For validating the possible involvement in GBC, we further assessed its expression in 36 pairs of fresh GBC tissues and CNG tissues. Indeed, reduced miR-218-5p expression was detected in GBC tumors samples (Figures 1c and d). In situ hybridization (ISH) staining also confirmed a remarkably lower miR-218-5p expression in GBC paraffin sections (Figure 1e). Moreover, we also noticed a reduced miR-218$5 p$ expression in different GBC cell lines compared with normal gallbladder epithelial cell (GBE) cells (Figure 1f). Intriguingly, further correlation analysis manifested no obvious relationship between miR-218-5p expression and patients' clinic characteristics such as TNM stage, tumor size, CA 19-9 level, gallstone status, age, and gender (Figure 1g; Supplementary Table 2). However, an inverse relationship between miR-218-5p and cumulative survival rate was observed (Figure 1h). 

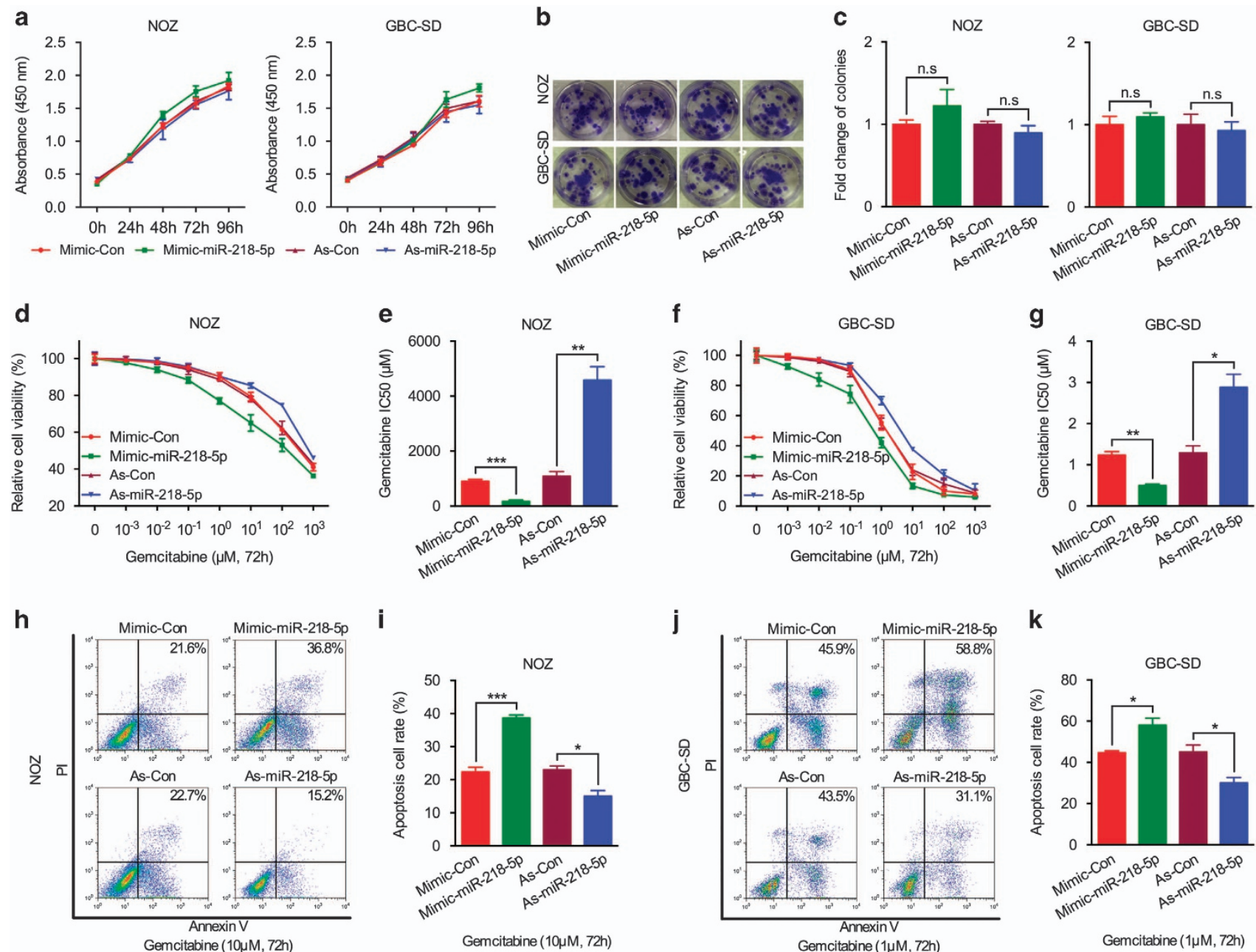

$\mathbf{k}$

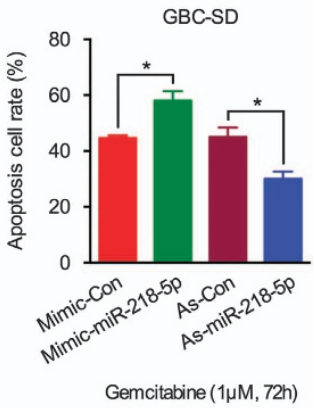

Figure 2 miR-218-5p promotes sensitivity of gemcitabine in NOZ and GBC-SD cell lines. (a) MTS analysis of GBC cells (NOZ and GBC-SD) transiently transfected with miR-218-5p mimic (Mimic-miR-218-5p), negative control mimic (Mimic-Con), miR-218-5p antisense (As-miR-218-5p), or negative control antisense (As-Con). (b-c) Representative images of colony formation and the mean fold change of colonies induced by miR-218-5p mimic or antagomir in GBC cells. (d-g) MTS assay and calculating the corresponding IC50 of gemcitabine in GBC cells transfected with miR-218-5p mimic or antagomir. (h-k) Flow cytometric analysis of Annexin V/PI staining used to quantify apoptosis rate of GBC cells transfected with miR-218-5p mimic or antagomir, followed by exposure to gemcitabine (NOZ, $10 \mu \mathrm{M}$; GBC-SD, $1 \mu \mathrm{M})$ for $72 \mathrm{~h}$. All $n=3$; bar, S.E.M., NS, not significant, ${ }^{*} P<0.05 ;{ }^{* *} P<0.01 ;{ }^{* *} P<0.001$; Student's t-test

Enforcing miR-218-5p expression accelerated tumor cell death and improved chemotherapeutic efficacy. To further explore the biological effect of miR-218-5p, we overexpressed and reduced miR-218-5p in GBC cell lines (NOZ and GBC-SD) by transfecting miR-218-5p mimics and antisense, respectively. However, no obvious difference in proliferation and colony formation ability was found (Figures $2 a-c$ ). GBC is well known for its higher chemoresistance than other kinds of tumors. Therefore, we wondered if miR-218-5p expression influences GBC patients' prognosis through influencing the chemotherapeutic efficacy. To verify this hypothesis, we treated miR-218-5p overexpressing or downregulated NOZ and GBC-SD cell lines with various concentrations of gemcitabine and analyzed the viability at $72 \mathrm{~h}$ post treatment. Surprisingly, enforced miR-218-5p expression improved the antitumor effect of gemcitabine in NOZ and GBC-SD cells, with a significant reduction in IC50. In contrast, reduced miR-218-5p expression enhanced gemcitabine resistance ability, with a remarkable increase in IC50 (Figures 2d-g). To validate these results, the effect of miR-218-5p was examined by flow cytometry analysis of Annexin $\mathrm{V}$ positive cells after gemcitabine addition. Consistently, similar results were obtained showing that miR-218-5p overexpression greatly accelerated cell death, and downregulation inhibited cell apoptosis after gemcitabine treatment (Figures 2h-k). However, miR-218-5p itself has no effect on the apoptosis of GBC cells (Supplementary Figure 3). In conclusion, our results demonstrate that miR-218-5p can sensitize GBC cells to chemotherapeutic treatment.

miR-218-5p targets $3^{\prime}$-UTR of PRKCE and suppresses its expression. miRNA usually regulate specific gene expression by binding the $3^{\prime}$-UTR of their target genes. Through prediction analysis using six different databases, two common targets of miR-218-5p were found (PRKCE and SFMBT1) (Figure 3a). We then analyzed the mRNA 
a

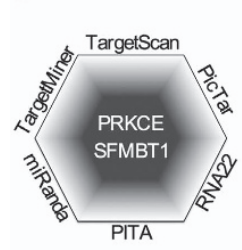

b

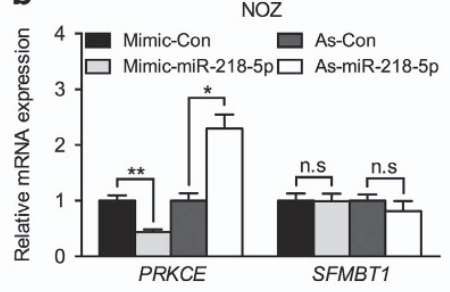

C

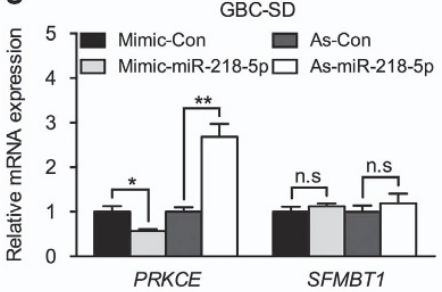

f

e

PRKCE-WT 5'-..CUGGAGUUGAGUACAAGCACAG..-3' miR-218-5p 3'-..UGUACCAAUCU AG-UUCGUGUU..-5

PRKCE-MU 5'-..CUGGAGUUGAGUACATC CTGAGG..-3' miR-218-5p 3'-..UGUACCAAUCU AG-UUCGUGUU...-5'

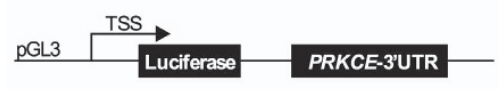

h

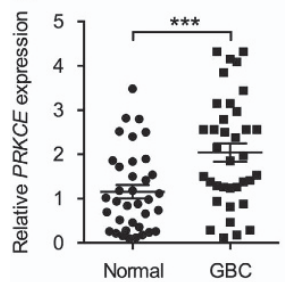

i

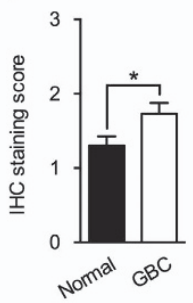

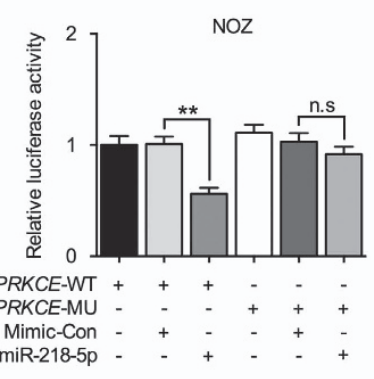

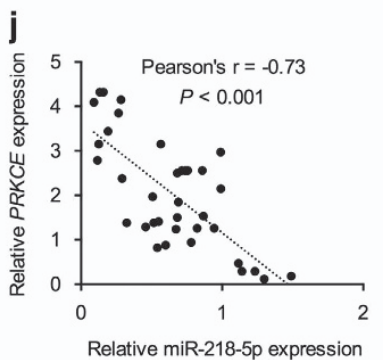

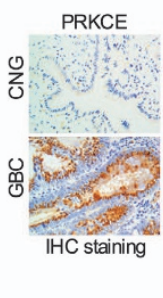

d

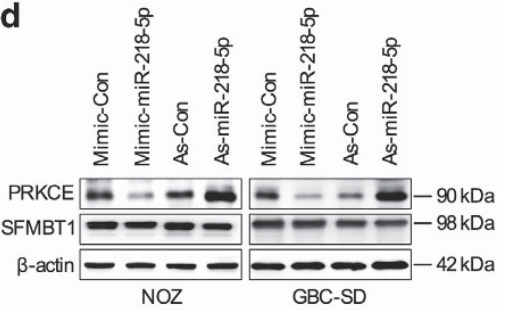

g

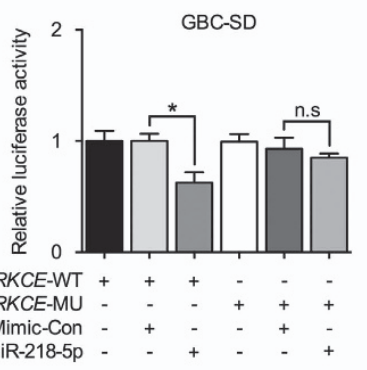

k

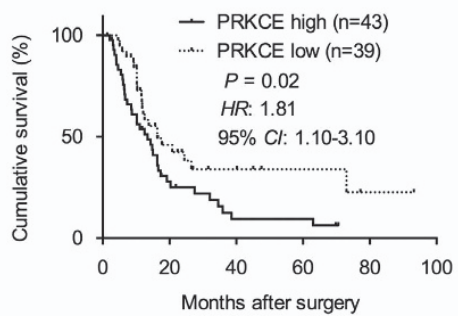

Figure 3 miR-218-5p directly targets the $3^{\prime}$-UTR of PRKCE and downregulated its expression. (a) PRKCE and SFMBT1 were potential targets of miR-218-5p in all six miRNA target prediction algorithms. (b, c) Q-PCR to detect PRKCE and SFMBT1 mRNA level in GBC cells when transfected with miR-218-5p mimic or antagomir. $n=3$; bar, S.E.M. (d) Western blot to analyze PRKCE and SFMBT1 protein levels in GBC cell lines when transfected with miR-218-5p mimic or antagomir. (e) A schematic diagram showing the predicted miR-218-5p binding sites and the designed mutant sequence in the $3^{\prime}-U T R$ of PRKCE (up), and the luciferase reporter constructs (down). (f $\mathbf{g}$ ) Firefly luciferase activity analysis of $P R K C E 3^{\prime}$-UTR performed after co-transfection with PRKCE-wild type or PRKCE-mutant pGL3 constructs and miR-218-5p mimic GBC cell lines. $n=3$; bar, S. E.M. (h) Q-PCR analysis of PRKCE mRNA levels in 36 pairs of GBC and CNG tissues. (i) Semi-quantitative analysis and the representative images ( $\times 400$ ) of IHC staining for $P R K C E$ protein in 82 paired GBC and CNG FFPE tissues. (j) The correlation between miR-218-5p and PRKCE expression in 36 GBC tissues measured by Q-PCR. (k) POS analysis based on PRKCE protein expression levels in $82 \mathrm{GBC}$ patients. GAPDH was used to normalize the Q-PCR results, and $\beta$-actin was the loading control in western blot assay. NS, not significant, ${ }^{\star} P<0.05 ;{ }^{* \star} P<0.01 ;{ }^{* \star *} P<0.001$; Student's $t$-test

expression of PRKCE and SFMBT1 in miR-218-5p mimics or antisense-transfecting NOZ and GBC-SD cells. Surprisingly, only PRKCE showed reduced expression in miR-218-5p overexpressing cells, and increased expression upon miR-218-5p downregulation (Figures $3 b$ and $c$ ). No obvious alterations in SFMBT1 mRNA expression were observed (Figures $3 \mathrm{~b}$ and $\mathrm{c}$ ). Further, we analyzed PRKCE and SFMBT1 protein expression by immunoblotting analysis, and found that miR-218-5p reduced PRKCE protein expression in both NOZ and GBC-SD cells. No obvious alterations in the SFMBT1 protein amount were detected (Figure 3d). We then mutated the predicted complementary paring region of the 3'-UTR of PRKCE-WT (5'-AAGCACA-3') to PRKCEMU (5'-ATCCTGA-3') (Figure 3e), and cloned into luciferase reporter vector. miR-218-5p addition reduced the luciferase activity carrying PRKCE-WT reporter but failed to do so with $P R K C E-M U$ transfection in NOZ cells (Figure 3f). Similar results were obtained in GBC-SD cells (Figure $3 \mathrm{~g}$ ). PRKCE has been previously suggested to be involved in multiple kinds of tumors, but its involvement in GBC has never been investigated. We analyzed PRKCE expression in our 36 pairs of $\mathrm{CNG}$ and $\mathrm{GBC}$ tumor samples, and found an increased expression in GBC (Figure 3h). Immunohistochemistry (IHC) staining further corroborated our results (Figure $3 \mathrm{i}$ ). Interestingly, an inverse correlation between miR-218-5p and PRKCE mRNA expression was detected in GBC samples (Figure 3j). Moreover, higher PRKCE expression correlated with reduced cumulative survival rate, implying a poorer prognosis with PRKCE overexpression (Figure 3k).

The chemotherapeutic sensitizer function of miR-218-5p is PRKCE dependent. To find out whether PRKCE is involved in regulating chemosensitivity of $\mathrm{GBC}$, we overexpressed and knocked down PRKCE in GBC cell lines using overexpression vector and siRNA, respectively. QuantitativePCR (Q-PCR) and immunoblotting analysis confirmed the overexpression and knock down efficiency of PRKCE (Figures $4 \mathrm{a}-\mathrm{d}$ ). Indeed, downregulation of PRKCE expression reduced IC50 of gemcitabine and increased tumor-killing effect of gemcitabine in NOZ and GBC-SD cells. On the other hand, PRKCE overexpression had the opposite effect and remarkably increased IC50 of gemcitabine, and enhanced 
a
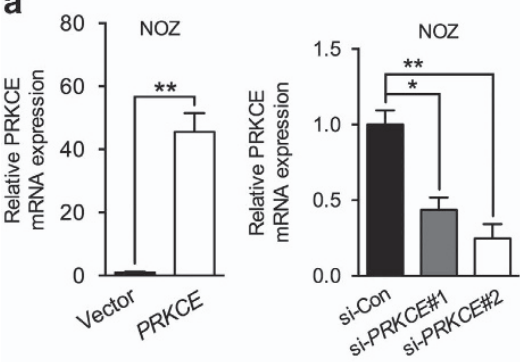

b

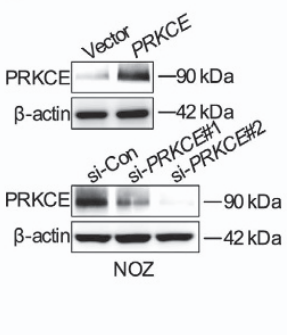

C

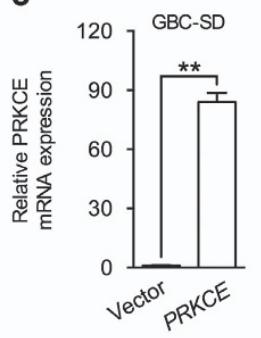

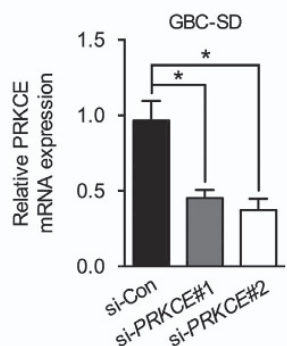

d

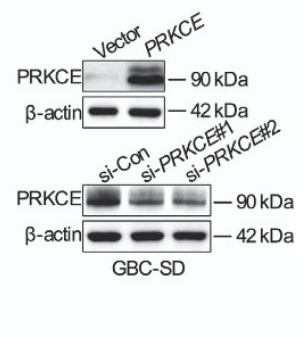

e

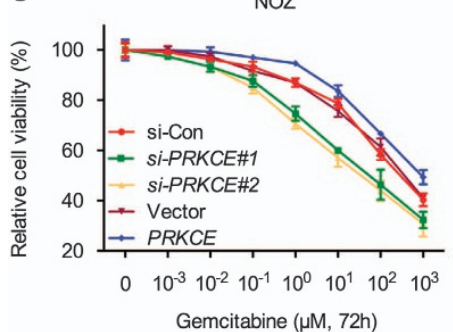

i

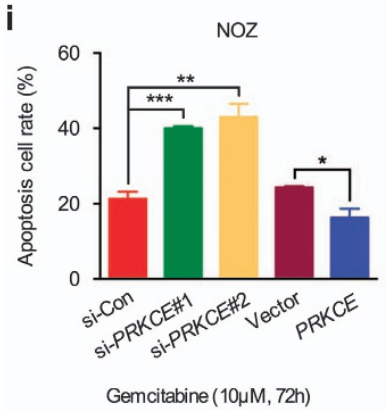

f
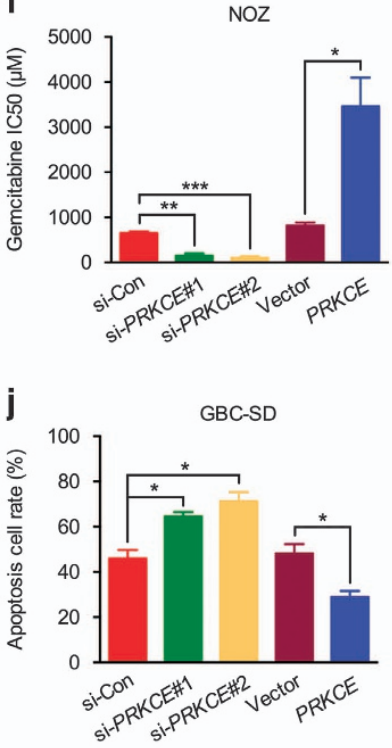

Gemcitabine $(1 \mu \mathrm{M}, 72 \mathrm{~h})$ g

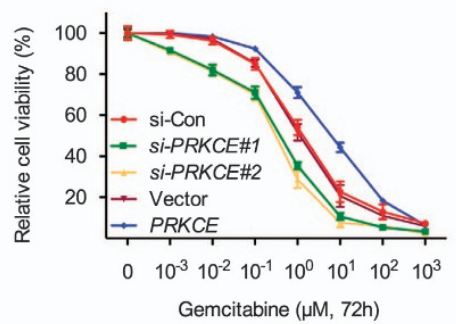

k

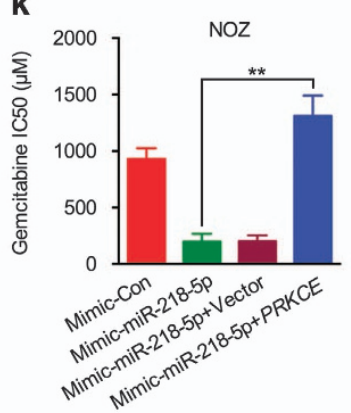

h
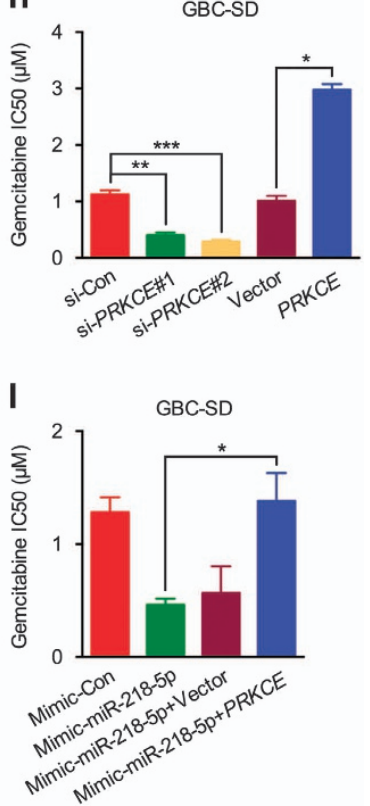

Figure 4 miR-218-5p-sensitized GBC cells to gemcitabine was mediated by PRKCE. (a-d) Efficiency testing of overexpression or interference of $P R K C E$ in GBC cells by using Q-PCR and western blot. (e-h) MTS assay and calculating the corresponding IC50 of gemcitabine in GBC cells transfected with PRKCE construct or PRKCE siRNAs. (i, j) Flow cytometric analysis of Annexin V/PI staining used to quantify apoptosis rate of GBC cells transfected with PRKCE construct or PRKCE siRNAs, followed by exposure to gemcitabine (NOZ, $10 \mu \mathrm{M}$; GBC-SD, $1 \mu \mathrm{M}$ ) for $72 \mathrm{~h}$. (k, I) IC50 of gemcitabine in GBC cells co-transfected with miR-218-5p mimic and PRKCE construct. GAPDH was used to normalize the Q-PCR results, and $\beta$-actin was the loading control in western blot assay. All $n=3$; bar, S.E.M. ${ }^{\star} P<0.05$; ${ }^{\star \star} P<0.01$; ${ }^{\star \star \star} P<0.001$; Student's $t$-test

chemoresistance (Figures $4 \mathrm{e}-\mathrm{j}$ ). Next for confirming PRKCE the potential downstream effector executing the anti-therapy effect with miR-218-5p deficiency, PRKCE were overexpressed in concomitant with miR-218-5p mimic transfection in $\mathrm{NOZ}$ and GBC-SD cell lines in parallel. As expected, PRKCE overexpression abolished the increased efficacy of gemcitabine with the addition of miR-218-5p mimic (Figures $4 k-1$ ). Our results demonstrate that miR-218-5p directly targeted $P R K C E$ and involved in the chemotherapeutic efficacy.

miR-218-5p/PRKCE targeted MDR1 involved in the chemoresistance of GBC. Our previously study showed that MDR1, MRP1, BCRP are involved in the chemoresistance mechanisms of GBC. ${ }^{4-6}$ Several studies have implicated PKC family in regulating several chemotherapeutic-resistant protein such as P-gp and BCRP. In order to find out whether miR-218$5 \mathrm{p}$ is also capable of modulating these proteins, MDR1, $M R P 1, B C R P$ mRNA, and protein expression were analyzed in NOZ and GBC-SD cells transfected with miR-218-5p mimic or antisense by Q-PCR and western blot. Among these three genes, only MDR1/P-gp exhibited an altered expression.
miR-218-5p overexpression inhibited MDR1/P-gp expression, while reduced miR-218-5p increased MDR1/P-gp expression (Figures $5 \mathrm{a}-\mathrm{d}$ ). On the other hand, our results also showed that MDR1/P-gp expression was regulated by PRKCE in NOZ and GBC-SD cells. Knockdown of PRKCE decreased MDR1/ P-gp levels, whereas overexpression of PRKCE increased MDR1/P-gp expression (Figures 5e-f). Interestingly, when PRKCE is overexpressed simultaneously with miR-218-5p mimic, MDR1/P-gp expression was restored (Figures $5 \mathrm{~g}-\mathrm{i}$ ). In addition, MDR1 overexpression not only strengthened the chemoresistance of GBC cells to gemcitabine, but also abrogated the effect of miR-218-5p overexpression on promoting gemcitabine sensitivity as shown by restored IC50 (Figure $5 \mathrm{j}$ ). All the above results demonstrated that miR-218$5 \mathrm{p}-P R K C E-M D R 1$ axis is a potential candidate target in GBC chemotherapeutic treatment.

The improved chemotherapeutic efficacy of miR-218-5p addition in vivo. In order to confirm the relationship of miR-218-5p with chemotherapeutic effect in vivo, we utilized a tumor xenograft mouse model. NOZ cells were stably 
a

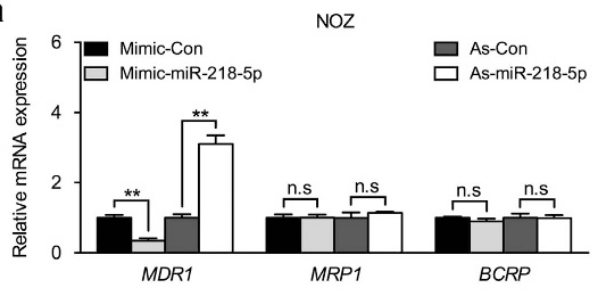

b
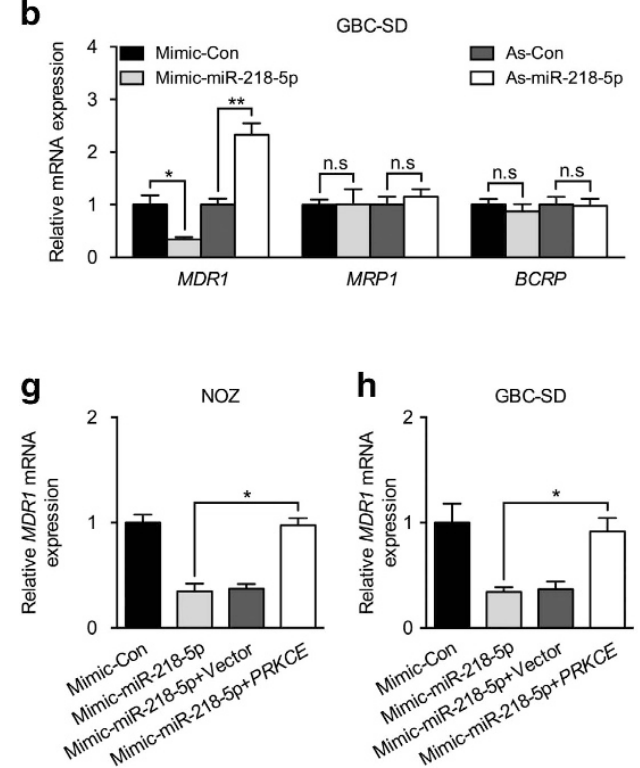

C

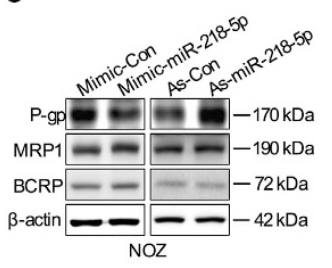

d

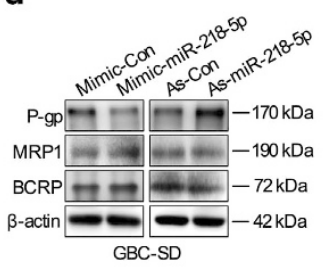

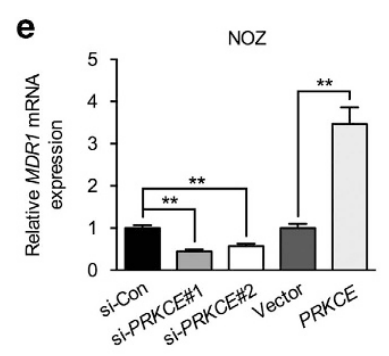

f
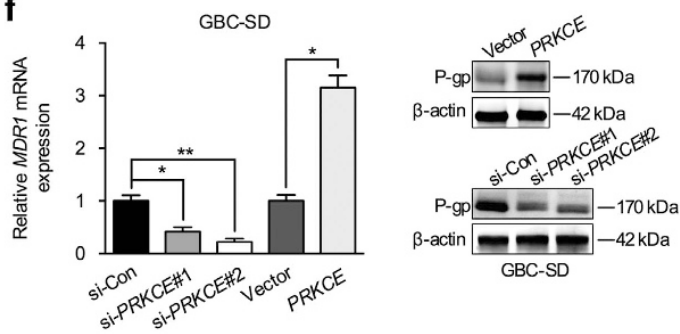

j
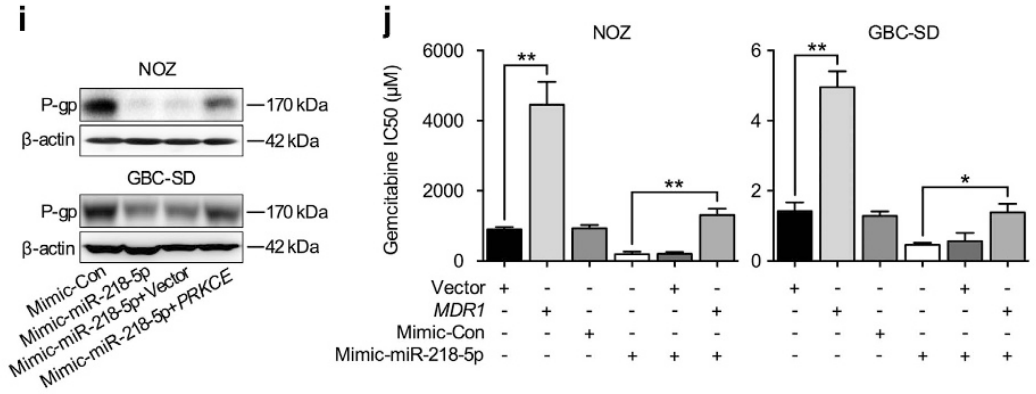

Figure 5 miR-218-5p negatively regulates resistance to gemcitabine through PRKCE/MDR1 axis in GBC cells. (a-d) Q-PCR and western blot to analyze MDR1, MRP1, and $B C R P$ mRNA and protein expression, respectively, in GBC cell lines when transfected with miR-218-5p mimic or antagomir. (e, f) Q-PCR and western blot to analyse MDR1 mRNA and P-gp expression, respectively, in GBC cell lines when transfected with PRKCE construct or PRKCE siRNAs. (g-i) Q-PCR and western blot to detect MDR1 mRNA and P-gp expression, respectively, in GBC cell lines when co-transfected with miR-218-5p mimic and PRKCE construct. (j) IC50 of gemcitabine in GBC cells when co-transfected with miR-218-5p mimic and MDR1 construct. GAPDH was used to normalize the Q-PCR results, and $\beta$-actin was the loading control in western blot assay. All $n=3$; bar, S.E.M. ${ }^{*} P<0.05 ;{ }^{* *} P<0.01$; Student's t-test

transfected with a miR-218-5p plasmid construct or control plasmid and subcutaneously injected into male nude mice. A week later, one group was treated with gemcitabine $(15 \mathrm{mg} / \mathrm{kg}$ per week) administered by intraperitoneal administration, and the other with the carrier (Saline). Consistently, miR-218-5p addition had no effect on tumor size in mouse group with no drug treatment (Figures $6 \mathrm{a}-\mathrm{c}$ ). However, tumor sizes and weight were greatly reduced in miR-218-5p overexpression mouse treated with gemcitabine (Figures $6 a-c)$. IHC analysis of PRKCE and P-gp both exhibited reduced expression with miR-218-5p overexpression (Figures 6d-e, g). Transferase dUTP nick end-labeling (TUNEL) analysis of tumor specimens showed that the antitumor effect of gemcitabine was greatly enhanced in miR-218-5p overexpression mouse due to increased apoptosis (Figures $6 \mathrm{f}-\mathrm{g}$ ). These results from tumor xenograft mouse model provided further evidence that miR-218-5p enhances gemcitabine-induced apoptosis by targeting PRKCE and MDR1.

\section{Discussion}

The high chemoresistance of GBC made its treatment even more difficult and prognosis more pessimistic. Our study identified a novel miR-218-5p-PRKCE-MDR1 pathway implicated in modulating GBC chemosensitivity (Figure 6h). Reduced miR-218-5p expression in GBC, disabled its ability in degrading PRKCE, which eventually led to increased MDR1/P-gp expression. P-gp, one of the MDR-related proteins, played important role in orchestrating chemoresistant effect of gemcitabine. ${ }^{19,20}$ Thus, future treatment targeting miR-218-5p-PRKCE-MDR1 pathway would provide promising prospect for GBC patients.

Increasing evidence suggests that miR-218-5p exhibits antitumor effect in the development and progression of various human cancers through influencing tumor proliferation, invasion, and metastasis progression. ${ }^{11-13,21}$ Moreover, recent studies suggest that miR-218-5p also exert critical role on regulating chemosensitivity. ${ }^{22-24}$ For example, miR-218-5p improved 5-fluorouracil therapeutic efficacy through inhibiting BIRC5 and TS expression, both of which are important contributors to chemoresistance. ${ }^{22}$ Another investigation in glioblastoma multiforme cells found that elevating miR-218-5p increased sensitivity to cisplatin via inactivating RTK-HIF2a signaling axis. ${ }^{23}$ More remarkably, altered miR-218-5p expression in breast cancer cells modulated the sensitivity of cisplatin through regulating BRCA1 expression. ${ }^{24}$ However, whether 


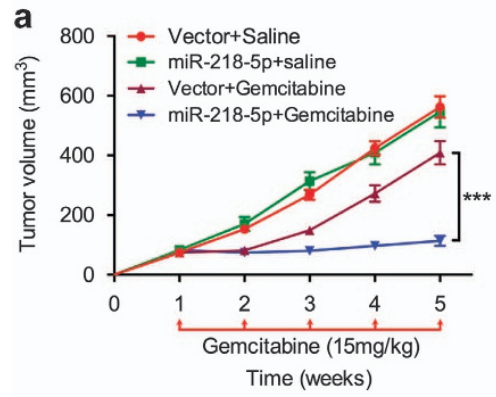

b
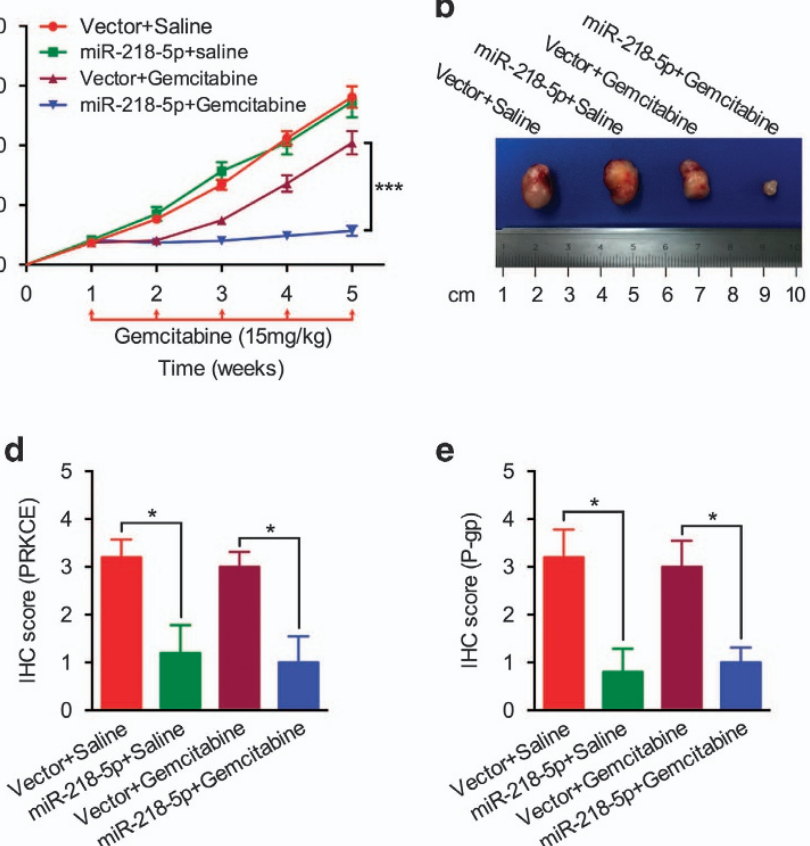

e

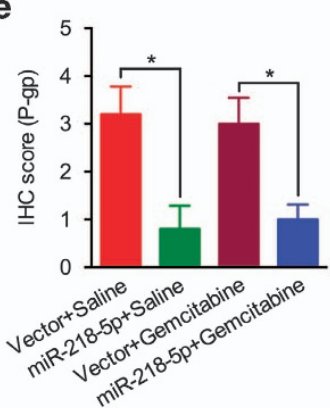

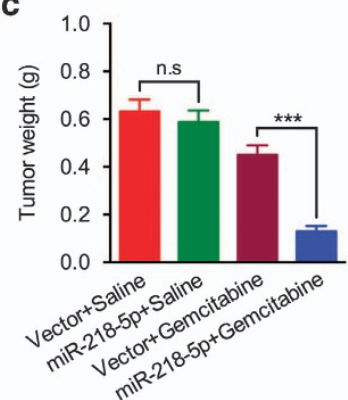

f

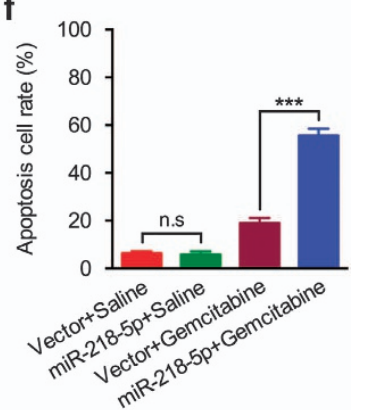

h

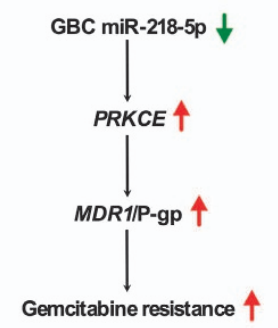

Downregulation $\downarrow \quad$ Upregulation $\uparrow$

Figure 6 Effect of miR-218-5p overexpression on the gemcitabine sensitivity of GBC cells in vivo. (a) Tumor growth curves of NOZ cells transfected with miR-218-5p construct or empty vector treated with gemcitabine or saline in vivo. (b, c) Representative images and the mean tumor weight of the four paired groups formed at the 5th week after subcutaneous transplantation. (d-f) Semi-quantitative analyses of IHC results for PRKCE and P-gp expression, and percentage of apoptotic tumor cells from the four paired NOZ tumor xenografts. (g) Representative images of PRKCE and P-gp IHC staining, and TUNEL staining in paraffin sections from the four paired NOZ tumor xenografts ( $\times 400)$. (h) Schematic representation of pathway modulated by miR-218-5p in GBC cells. Downregulation of miR-218-5p in GBC cells can elevate PRKCE expression. This increases the expression of MDR1/P-gp, resulting in gemcitabine resistance of GBC. All $n=5$; bar, S.E.M. NS, not significant, ${ }^{\star} P<0.05 ;{ }^{* \star *} P<0.001$; Student's $t$-test

and how miR-218-5p might be involved in GBC has not been studied. Consistent with previous studies, we also found a significantly reduced miR-218-5p expression of GBC tissues compared with CNG tissues and correlated with a poor prognosis. Simultaneously, an increased sensitivity to gemcitabine was observed in vitro miR-218-5p overexpression. But no obvious effects on GBC cell growth and apoptosis were detected. This implied that miR-218-5p specifically influenced GBC chemosensitivity and thus changed GBC patients' prognosis.

So far, miR-218-5p has been reported to bind to various targets such as ARAF, PIK3C2A, LASP1, BRCA1, SFMBT1, DCUN1D1, and $P X N$ to repress corresponding gene translation. ${ }^{12,13,23-26}$ However, the function of miR-218-5p and the association of its target gene with gemcitabine resistance in GBC remains unclear. Further elucidating the precise mechanism inside, in silico analysis of putative miRNA-binding sites in $3^{\prime}$-UTR of the target genes by using different algorithms (TargetScan, PicTar, RNA22, PITA, miRanda, and TargetMiner) was performed. PRKCE and SFMBT1 were finally identified, both harboring evolutionarily conserved targeting sequence of miR-218-5p. Facilitated by further in vitro overexpression and site mutation-related luciferase assay, we then confirmed the regulatory role of PRKCE by miR-218-5p in GBC tumors. Emerging evidences suggested that $P R K C E$ plays oncogenic role through increasing survival protein levels, which sustained cancer stem cell development. ${ }^{17}$ Our study reinforced the oncogenic role of $P R K C E$ in increasing gemcitabine resistance and its correlation with a poor prognosis in GBC tumors. The inverse relationship between miR-218-5p and PRKCE was then confirmed in GBC tissues. Therefore, early examination of miR-218-5p and PRKCE expression in the tumors of GBC patients may aid in the prediction of chemotherapeutic effect and help in the development of new postoperative chemotherapy strategies. However, preclinical and clinical studies with 
large sample sizes and longer follow-up times are required to confirm the utility of these potential biomarkers.

Reducing intracellular drug accumulation is one of the most important mechanisms utilized by cancer cells in chemoresistance. Elevated drug efflux pump-related proteins such as P-gp, MRP1, MRP2, and BCRP expression significantly promotes cancer chemoresistance. ${ }^{27}$ Consistent with the previous report in renal carcinoma cells that reduce the expression of PRKCE lowered MDR1/P-gp, which affects the cancer stem cell potential of sorted side population cells and suppresses proliferation potential, resistance to chemotherapeutics and in vivo tumor formation ability. ${ }^{17}$ Our identification of the regulatory role of miR-218-5p-PRKCEMDR1 axis in GBC cells further confirmed its importance.

Taken together, our study demonstrated that miR-218-5p is a potent tumor chemoresistance suppressor in the GBC and that its chemosensitization effects are mediated, at least in part, via downregulation of the PRKCE/MDR1 pathway. Loss of miR-218-5p expression, leading to the induction of $P R K C E$ and its downstream MDR1/P-gp expression, appears to be a critical event in the development of gemcitabine resistance.

\begin{abstract}
Materials and Methods
Patients and tissue samples. This study was approved by the Ethical Committee of Renji hospital, Shanghai Jiao Tong University School of Medicine. A total of 82 pairs of formalin-fixed, paraffin-embedded (FFPE) GBC tissues and CNG tissues were retrieved from $\mathrm{GBC}$ patients who underwent surgical resection and postoperative adjuvant chemotherapy from the Department of Pathology of Renj Hospital. Among the 82 GBC patients, 36 pairs of fresh GBC tissues and CNG tissues were also collected after the surgical removal and snap-frozen in liquid nitrogen immediately, then stored at $-80^{\circ} \mathrm{C}$ until total RNA was extracted. All 82 GBC patients were retrospectively followed up until December 2014. Postoperative survival (POS) was defined as the interval between the dates of surgery and last follow-up or death. All the subjects provided written informed consent in this study.
\end{abstract}

Cell culture. Three different kinds of human GBC cell lines were used in this study. GBC-SD was purchased from the Cell Bank of Type Culture Collection of Chinese Academy of Sciences (Shanghai, China). SGC-996 was provided by the academy of life sciences, Tong Ji university (Shanghai, China). NOZ were obtained from the Health Science Research Resources Bank (Osaka, Japan). GBC-SD, SGC-996, and NOZ were cultured in DMEM, RPMI-1640, and William's E medium (Gibco, Grand Island, NY, USA), respectively, which supplemented with $10 \%$ fetal bovine serum (FBS) in a humidified atmosphere of $5 \% \mathrm{CO}_{2}$ at $37^{\circ} \mathrm{C}$. All cell lines were ensured mycoplasma-negative cultures by monthly mycoplasma tests. For primary GBE culturing, cells from digested gallbladder epithelial tissue were cultured in KSFM medium without FBS. After $30 \mathrm{~min}$, adherent cells were discarded, and non-adherent cells were continually cultured at $37^{\circ} \mathrm{C}$ in $5 \%$ humidified $\mathrm{CO}_{2}$.

Cell transfection. The miR-218-5p mimic and a non-specific mimic control, miR-218-5p antisense and a non-specific antisense control, PRKCE siRNA, and a negative control siRNA were all purchased from GenePharma (Shanghai, China), and were transfected into GBC cell lines by using Lipofectamine 2000 reagent (Invitrogen, Carlsbad, CA, USA) according to instructions of the manufacturer. Human miR-218$5 p$ expression construct was generated by insertion of the coding sequence (CDS) of miRNA into pCDH-CMV-MCS-EF1-copGFP (System Biosciences, Palo Alto, CA, USA). Then recombinant lentiviruses produced in HEK293FT cells infect GBC cell lines in the presence of $4 \mu \mathrm{g} / \mathrm{ml}$ polybrene (Sigma, St. Louis, MO, USA), followed by puromycin selection $(2 \mu \mathrm{g} / \mathrm{ml})$ for stably overexpressing GBC cell lines. PRKCE and $M D R 1$ overexpression vector was generated by insertion of gene CDS into a pcDNA 3.1 vector (Invitrogen). GBC-SD and NOZ cells were transfected with the overexpression plasmid using Lipofectamine 2000 reagent. The PCDH-CMV-MCSEF1-copGFP and pcDNA 3.1 empty vector were used as negative control (vector), respectively.
Cloning efficiency assays. For colony formation, cells in single-cell suspension were plated and grown in 24-well plates at a density of 100 per well for $24 \mathrm{~h}$. After transfecting, GBC cells were incubated in the plate for 14 days until colonies were visible. The colonies were fixed for $15 \mathrm{~min}$ with $4 \%$ paraformaldehyde and stained with $0.1 \%$ crystal violet. The numbers of colonies were counted to assess the viability.

Cell viability and apoptosis assays. After transfecting with miRNA mimic or antisense and treating with concentration gradient of gemcitabine (Selleck, Houston, TX, USA), the viability of GBC cell was measured using 3-(4,5-dimethylthiazol-2-yl)-5(3-carboxymethoxyphenyl)-2-(4-sulfophenyl)-2H-tetrazolium (Promega, Madison, WI, USA) assay (MTS), as described previously. ${ }^{4}$ After transfecting with gemcitabine for $48 \mathrm{~h}$, cells were stained with FITC-conjugated Annexin V (BD Biosciences, Heidelberg, Germany) and propidium iodide ( $5 \mathrm{mg} / \mathrm{ml})$ and analyzed by fluorescence-activated cell sorting analysis, as described previously. ${ }^{4}$

Immunoblot analysis. Total proteins were extracted from GBC cells using cell lysates in RIPA buffer and protein expression was assessed by immunoblot analysis according to the procedure described previously. The primary mouse, rabbit, or goat antibodies used were as follows: PRKCE (1:500, sc-1681, Santa Cruz, Santa Cruz, CA, USA), SFMBT1 (1:500, ab77419, Abcam, Cambridge, UK), MDR1/P-gp (1:500, sc-55510, Santa Cruz), MRP1 (1:2000, sc-365635, Santa Cruz), BCRP (1:200, sc25822, Santa Cruz), and $\beta$-actin (1:2000, A5316, Sigma).

Quantitative real-time PCR analysis. TRI reagent (Sigma) was used to isolate total RNA for mRNA analysis, and miRNeasy kit (Qiagen, Valencia, CA, USA) for miRNA analysis from the snap-frozen tissues or cultured cells. After determining RNA concentration and purity by using NanoDrop ND-8000 (Thermo Fisher Scientific, Waltham, MA, USA), the CDNAs were synthesized by using Reverse Transcriptase M-MLV kit (Invitrogen). The expression levels of miRNA and mRNA were analyzed using SYBR Premix Ex Taq (Takara, Shiga, Japan) in Applied Biosystems ViiATM 7 Real-Time PCR System (Applied Biosystems, Foster City, CA, USA). Data were analyzed with $2^{-\Delta \Delta C T}$ method ${ }^{28}$ for quantification of the relative mRNA expression levels. Expression values of genes and miRNA were first normalized against GAPDH and small nuclear U6 RNA, and then compared to experimental controls. The primers were purchased from Sangon Biotech (Shanghai, China) and the sequences are listed in Supplementary Table 3.

Immunohistochemistry and terminal deoxynucleotidyl transferase dUTP nick end-labeling assays. The IHC and TUNEL staining of FFPE tissue and its scoring system were performed, as described previously. ${ }^{4}$ The mouse primary antibodies used were as follows: PRKCE (1:200, sc-1681, Santa Cruz), MDR1/ P-gp (1:200, sc-55510, Santa Cruz).

Dual-luciferase reporter assay. The $3^{\prime}$-UTR of PRKCE containing the predicted miR-218-5p binding site was amplified by PCR using CDNA of GBC cell as a template and then subcloned into a pmirGLO Dual-Luciferase miRNA Target Expression Vector (Promega) to form the reporter vector MRP1-3'-UTR wild type. The mutant $3^{\prime}$-UTR of PRKCE, which contained point-mutated sequence in the binding region of miR-218-5p, was generated by using a site-directed mutagenesis kit from Fast Mutagenesis System (TransGen Biotech, Beijing, China). NOZ and GBC-SD cells were seeded into 96-well plates $\left(1 \times 10^{4}\right.$ cells per well) and then cotransfected with miR-218-5p mimics and the luciferase reporter construct. After transfection for $48 \mathrm{~h}$, the Renilla and firefly luciferase activities were analyzed using the Dual-Luciferase Assay System (Promega). The results of firefly luciferase activity were normalized to the Renilla luciferase activity.

Bioinformatics. The target genes of miR-218-5p were predicted by six computer-aided algorithms, ${ }^{29-32}$ namely TargetScan Release 7.0 (http://www. targetscan.org/vert_71/), PicTar (http://www.pictar.org/cgi-bin/new_PicTar_vertebrate. cgi), RNA22 (https://cm.jefferson.edu/rna22/), PITA (https://genie.weizmann.ac.il/pubs/ mir07/mir07_prediction.html), miRanda (http://www.microrna.org/microrna/home.do), and Targetminer (http://www.isical.ac.in/ bioinfo_miu/targetminer20.htm). The target genes were accepted only when they were positive in all six analyses.

In situ hybridization. ISH of GBC FFPE tissues were performed using a human miR-218-5p-specific digoxigenin-labeled locked nucleic acid probe. Briefly, following dewaxing and rehydration, tissue sections were treated with proteinase $\mathrm{K}$ at $37^{\circ} \mathrm{C}$ for $15 \mathrm{~min}$. We then washed specimens with PBS and dehydrated through a graded series of ethanol $(70,96$ and $100 \%)$. After incubation under $50{ }^{\circ} \mathrm{C}$ 
overnight with miR-218-5p probe in hybridization buffer, the slides were washed in pre-warmed $5 \times, 1 \times$ and $0.2 \times \mathrm{SSC}$ at $50^{\circ} \mathrm{C}$ for $30 \mathrm{~min}$. The slides were blocked with blocking reagent (Roche, Mannheim, Germany) and then incubated with antibody against digoxigenin (1:1000, Roche) at room temperature for $30 \mathrm{~min}$, respectively. Finally, the substrate NBT/BCIP (Roche) was added on specimens and incubated for $15 \mathrm{~min}$ in dark until the specific blue signal was observed followed by stopping further reaction using KTBT buffer.

Tumor formation assay in a nude mouse model. Twenty male BALB/C nude mice (4 weeks old; $15-25 \mathrm{~g}$ ) were randomly divided into two groups. A total $2 \times 10^{6} \mathrm{NOZ} / \mathrm{pCDH}$ empty vector and NOZ/pCDH miR-218-5p overexpression cells in $100 \mu \mathrm{l}$ medium were subcutaneously transplanted into the mouse left flank of two groups, respectively. One week later, each group was randomly divided into two subgroups $(n=5)$ and subjected to intraperitoneal injection of gemcitabine (15 mg/ $\mathrm{kg}$ ) or saline (100 $\mu \mathrm{l}$; negative control) weekly. Tumor growth was measured using external caliper once every week and were calculated based on the equation: $V=\left(\right.$ length $\times$ width $\left.^{2}\right) / 2 .^{33}$ All mice were killed at the 5 th week, and the tumors were dissected out for IHC and TUNEL staining. The study was strictly performed accordance with the recommendations in the Guide for the Care and Use of Laboratory Animals of Shanghai Jiao Tong University.

Statistics. Data were expressed as mean \pm S.E.M. Group comparisons of normally distributed data were performed with unpaired Student's t-test (two-tailed) or one-way ANOVA. For multiple comparisons, the Tukey-Kramer honestly significant difference was applied following ANOVA. Kaplan-Meier analyses log-rank test were used to determined POS. The Pearson $\chi^{2}$-test was used to analyze the association of miR-218-5p expression with PRKCE expression. Dichotomous variables were compared using $\chi^{2}$-test. SPSS17.0 software (IBM, Chicago, IL, USA) was used for all statistical analysis. $P<0.05$ was considered statistically significant.

\section{Conflict of Interest}

The authors declare no conflict of interest.

Acknowledgements. This work was supported by the National Science Foundation of China (No. 81072011, 81272748 and 81472240 to JW), National Key Technology R\&D Program (No. 2012BAI06B01 to JW), Foundation of Science and Technology Commission of Shanghai Municipality (No. 12XD1403400 to JW), and Foundation of Shanghai Municipal Health Bureau (No. XBR2011035 to JW).

1. Castro FA, Koshiol J, Hsing AW, Devesa SS. Biliary tract cancer incidence in the United Statesdemographic and temporal variations by anatomic site. Int J Cancer 2013; 133: 1664-1671.

2. de Groen PC, Gores GJ, LaRusso NF, Gunderson LL, Nagorney DM. Biliary tract cancers. N Engl J Med 1999; 341: 1368-1378.

3. Lazcano-Ponce EC, Miquel JF, Munoz N, Herrero R, Ferrecio C, Wistuba Il et al. Epidemiology and molecular pathology of gallbladder cancer. CA Cancer J Clin 2001; 51: 349-364.

4. Zhan M, Wang H, Chen T, Chen W, Yang L, He M et al. NOX1 mediates chemoresistance via HIF1alpha/MDR1 pathway in gallbladder cancer. Biochem Biophys Res Commun 2015; 468: 79-85.

5. Zhan M, Zhao X, Wang H, Chen W, Xu S, Wang W et al. miR-145 sensitizes gallbladder cancer to cisplatin by regulating multidrug resistance associated protein 1. Tumour Biol 2016; 37: 10553-10562.

6. Li XX, Dong Y, Wang W, Wang HL, Chen YY, Shi GY et al. Emodin as an effective agent in targeting cancer stem-like side population cells of gallbladder carcinoma. Stem Cells Dev 2013; 22: 554-566.

7. He L, Hannon GJ. MicroRNAs: small RNAs with a big role in gene regulation. Nat Rev Genet 2004; 5: 522-531.

8. Xuan Y, Yang H, Zhao L, Lau WB, Lau B, Ren N et al. MicroRNAs in colorectal cancer: small molecules with big functions. Cancer Lett 2015; 360: 89-105.

9. Chan B, Manley J, Lee J, Singh SR. The emerging roles of microRNAs in cancer metabolism. Cancer Lett 2015; 356(2 Pt A): 301-308.

10. Wu Q, Yang Z, Nie Y, Shi Y, Fan D. Multi-drug resistance in cancer chemotherapeutics: mechanisms and lab approaches. Cancer Lett 2014; 347: 159-166.

11. Ma MZ, Chu BF, Zhang Y, Weng MZ, Qin YY, Gong W et al. Long non-coding RNA CCAT1 promotes gallbladder cancer development via negative modulation of miRNA-218-5p. Cell Death Dis 2015; 6: e1583.
12. Martinez I, Gardiner AS, Board KF, Monzon FA, Edwards RP, Khan SA. Human papillomavirus type 16 reduces the expression of microRNA-218 in cervical carcinoma cells. Oncogene 2008; 27: 2575-2582.

13. Zhang X, Shi H, Tang H, Fang Z, Wang J, Cui S. miR-218 inhibits the invasion and migration of colon cancer cells by targeting the PI3K/Akt/mTOR signaling pathway. Int J Mol Med 2015; 35: 1301-1308.

14. Nishikawa R, Goto $Y$, Sakamoto S, Chiyomaru T, Enokida H, Kojima $S$ et al. Tumorsuppressive microRNA-218 inhibits cancer cell migration and invasion via targeting of LASP1 in prostate cancer. Cancer Sci 2014; 105: 802-811.

15. Gottesman MM, Fojo T, Bates SE. Multidrug resistance in cancer: role of ATP-dependent transporters. Nat Rev Cancer 2002; 2: 48-58.

16. Lau E, Sedy J, Sander C, Shaw MA, Feng Y, Scortegagna M et al. Transcriptional repression of IFN $\beta 1$ by ATF2 confers melanoma resistance to therapy. Oncogene $2015 ; 34: 5739-5748$.

17. Huang B, Fu SJ, Fan WZ, Wang ZH, Chen ZB, Guo SJ et al. PKCepsilon inhibits isolation and stemness of side population cells via the suppression of $\mathrm{ABCB} 1$ transporter and PI3K/Akt, MAPK/ERK signaling in renal cell carcinoma cell line 769 P. Cancer Lett 2016; 376: 148-154.

18. Griffiths-Jones S, Saini HK, van Dongen S, Enright AJ. miRBase: tools for microRNA genomics. Nucleic Acids Res 2008; 36: D154-D158.

19. Krech T, Scheuerer E, Geffers R, Kreipe H, Lehmann U, Christgen M. ABCB1/MDR1 contributes to the anticancer drug-resistant phenotype of IPH-926 human lobular breast cancer cells. Cancer Lett 2012; 315: 153-160.

20. Lee SW, Lee YL, Lee YJ, Park SY, Kim IS, Choi TH et al. Enhanced antitumor effects by combination gene therapy using MDR1 gene shRNA and HSV1-tk in a xenograft mouse model. Cancer Lett 2010; 291: 83-89.

21. Chiu KL, Kuo TT, Kuok QY, Lin YS, Hua CH, Lin CY et al. ADAM9 enhances CDCP1 protein expression by suppressing miR-218 for lung tumor metastasis. Sci Rep 2015; 5: 16426.

22. Liu Y, Cai Q, Bao PP, Su Y, Cai H, Wu J et al. Tumor tissue microRNA expression in association with triple-negative breast cancer outcomes. Breast Cancer Res Treat 2015; 152 183-191.

23. Mathew LK, Skuli N, Mucaj V, Lee SS, Zinn PO, Sathyan P et al. miR-218 opposes a critical RTK-HIF pathway in mesenchymal glioblastoma. Proc Natl Acad Sci USA 2014; 111: 291-296.

24. He X, Xiao X, Dong L, Wan N, Zhou Z, Deng $H$ et al. MiR-218 regulates cisplatin chemosensitivity in breast cancer by targeting BRCA1. Tumour Biol 2015; 36: 2065-2075.

25. Wu DW, Cheng YW, Wang J, Chen CY, Lee H. Paxillin predicts survival and relapse in nonsmall cell lung cancer by microRNA-218 targeting. Cancer Res 2010; 70: 10392-10401.

26. Jiang Z, Song Q, Zeng R, Li J, Li J, Lin X et al. MicroRNA-218 inhibits EMT, migration and invasion by targeting SFMBT1 and DCUN1D1 in cervical cancer. Oncotarget 2016; 7: 45622-45636.

27. Chen Z, Shi T, Zhang L, Zhu P, Deng M, Huang $C$ et al. Mammalian drug efflux transporters of the ATP binding cassette $(A B C)$ family in multidrug resistance: a review of the past decade. Cancer Lett 2016; 370: 153-164.

28. Livak KJ, Schmittgen TD. Analysis of relative gene expression data using real-time quantitative PCR and the 2(-Delta Delta C(T)) Method. Methods 2001; 25: 402-408.

29. Agarwal V, Bell GW, Nam JW, Bartel DP. Predicting effective microRNA target sites in mammalian mRNAs. Elife 2015; 4: e05005

30. Miranda KC, Huynh T, Tay Y, Ang YS, Tam WL, Thomson AM et al. A pattern-based method for the identification of microRNA binding sites and their corresponding heteroduplexes. Cell 2006; 126: 1203-1217.

31. Kertesz M, lovino N, Unnerstall U, Gaul U, Segal E. The role of site accessibility in microRNA target recognition. Nat Genet 2007; 39: 1278-1284.

32. Betel D, Koppal A, Agius P, Sander C, Leslie C. Comprehensive modeling of microRNA targets predicts functional non-conserved and non-canonical sites. Genome Biol 2010; 11: R90.

33. Tomayko MM, Reynolds CP. Determination of subcutaneous tumor size in athymic (nude) mice. Cancer Chemother Pharmacol 1989; 24: 148-154.

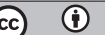

Cell Death and Disease is an open-access journal published by Nature Publishing Group. This work is licensed under a Creative Commons Attribution 4.0 International License. The images or other third party material in this article are included in the article's Creative Commons license, unless indicated otherwise in the credit line; if the material is not included under the Creative Commons license, users will need to obtain permission from the license holder to reproduce the material. To view a copy of this license, visit http://creativecommons.org/licenses/by/4.0/

C) The Author(s) 2017 